\title{
Microarrays in Parkinson's Disease: A Systematic Approach
}

\author{
Renee M. Miller* and Howard J. Federoff* ${ }^{\dagger}$ \\ *Center for Aging and Developmental Biology and ${ }^{\dagger}$ Department of Neurology, University of Rochester, Rochester, New York
}

Summary: Neurological disease (ND) is one of the greatest challenges facing our population, from medical, financial, and social perspectives. The application of new research approaches to understand the underlying pathogenesis of ND is critical. In this article, we review the use of microarray analysis in Parkinson's disease (PD). Microarrays have tremendous power, simultaneously querying the expression of tens of thousands of genes from a given biological sample. Coupled with impressive advances in statistical tools for analyzing large, complex data sets, well-designed microarray experiments are poised to make a big impact in the field of ND. Parkinson's disease is a devastating neurodegenerative disease well suited to a systems-based microarray analysis. Genetic and environmental rodent models of PD emulate many of the cardinal features of human PD, providing the unique opportunity to compare gene expression profiles from different etiologies of the same disease. The elucidation of important gene expression patterns during disease will make possible identification of genetic susceptibility markers, biomarkers of disease progression, and new therapeutic targets. Key Words: Microarray, Parkinson's disease, human, MPTP, $\alpha$-synuclein, gene expression.

\section{INTRODUCTION}

Neurological diseases (ND) pose significant health and financial challenges to individuals and society as a whole. Those conditions with age as a risk factor, such as Alzheimer's (AD) or Parkinson's disease (PD), are particularly confounding. The urgency to understand the causes and pathogenesis, as well as to develop better therapeutics for neurodegenerative diseases, is paramount. One key obstacle in this endeavor is the multifactorial nature of such diseases. It is widely believed that $\mathrm{PD}$ and $\mathrm{AD}$, for example, can be rarely caused by heritable genetic mutations in a small number of genes (e.g., $\alpha$-synuclein in PD) or environmental exposure to a selective toxin [e.g., 1-methyl-4-phenyl-1,2,3,6-tetrahydropyridine (MPTP) in PD], but most commonly result from a combination of genetic susceptibility factors and environmental conditions that remain extremely poorly understood. Because the genetic, environmental, and idiopathic cases of PD are clinically and neuropathologically so similar, we have hypothesized that a common pathological cascade of molecular events occurs in PD. ${ }^{1}$ In this review, we discuss the use of microarrays to try to

Address correspondence and reprint requests to: Howard J. Federoff, M.D., Ph.D., Director, Center for Aging and Developmental Biology, University of Rochester, 601 Elmwood Ave. Box 645, Rochester, NY 14642. E-mail: howard_federoff@urmc.rochester.edu. elucidate critical steps in the cascade. Special attention is given to a systems-based approach to analyzing gene expression in PD by comparing data from PD models and human patients at different disease stages and different brain regions. As more microarray data become available, we hope this analysis can be extended such that an accurate transcriptional phenotype of disease progression during PD is described.

Studies in $\alpha$-synuclein transgenic mice and MPTPtreated animals have been moderately successful in elucidating pathogenic pathways involved in PD. Significant advances include models to explain the unique susceptibility of dopaminergic (DA) neurons in $\mathrm{PD},{ }^{2-4}$ the role of glial factors in influencing neuronal decisions to live or die, ${ }^{5}$ the role of protein degradation or aggregation in disease, ${ }^{6-8}$ and many more. Model systems for Parkinson's disease were recently reviewed in more detail. ${ }^{9,10}$ Many of these studies are based on a candidate gene approach, in which the involvement of a particular protein in DA neuron pathology or death is ascertained via its genetic deletion or overexpression (FIG. 1A). This methodology is capable of describing important requirements at the single gene level for elements of the pathological cascade in a given setting, i.e., acute MPTP intoxication, and altered expression of the culprit gene may later be validated in postmortem human PD brain tissue. A candidate gene approach requires significant investment of time and resources but has the advantages of 
A.

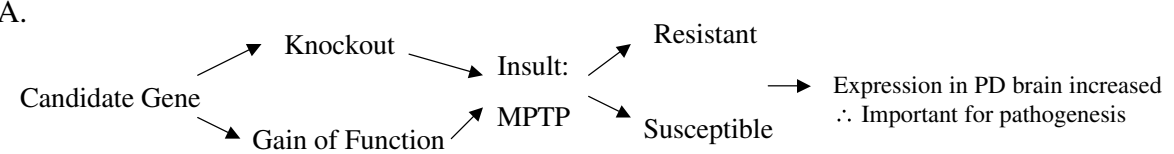

B.

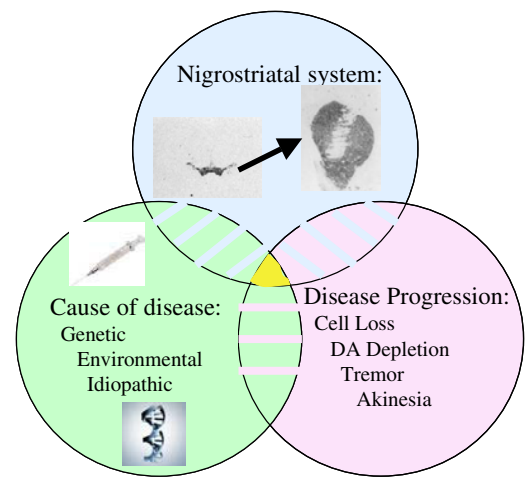

C.

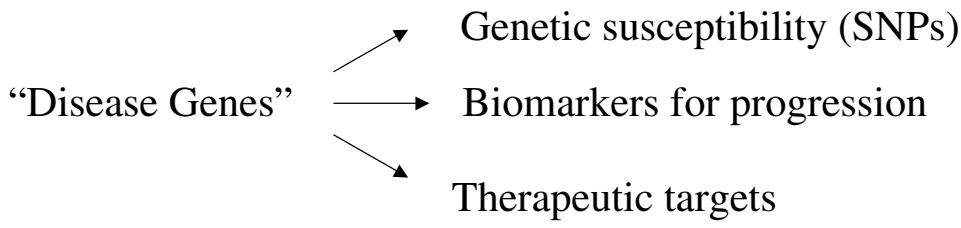

FIG. 1. Theoretical approaches to understanding pathogenesis in PD. To test the involvement of a specific candidate gene in the pathogenesis of PD-causing injury, MPTP in this example (A), the gene of interest may be deleted or overexpressed. If the gene's function is required for the evolution of injury and cell death, the knockout animal ought to be resistant to the insult, MPTP. On the other hand, excess gene product may exacerbate injury or enhance the susceptibility of normally resistant cell types. Observation of altered expression in the human postmortem brain from PD patients serves as further validation that the gene participates in pathogenesis. One can approach the same question from a wider perspective, asking which genes are differentially expressed in each brain region (blue), at each stage of disease (lavender), and in neurons exposed to different PD-causing insults (green) (B). Both unique and common gene expression patterns are expected within the realm of each circle, though points of overlap between circles are also of interest (hatched areas and yellow central area). Once identified, candidate disease genes are of use to both clinicians and researchers (C). The primary sequence can be analyzed for single nucleotide polymorphisms that may influence the genetic susceptibility of some portion of the population. Changes in expression of candidate genes may serve as markers of disease progression and the efficacy of therapeutic intervention. The overall goal is to generate novel targets for pathogenesis-directed therapies that will halt disease progression and ameliorate symptoms.

yielding readily interpretable data. In this review, we explore an alternate, system-based approach to understanding pathogenesis in ND, using PD and model systems of PD as examples (FIG. 1B).

What is a systems-based approach to understanding PD? The core elements of the system are DA neurons in the substantia nigra (SN) and target neurons in the striatum. Bidirectional communication between these neurons (ignoring other basal ganglia, thalamic, and cortical circuitry for simplicity) is critical to the proper execution of motor commands. During PD, one element of the system is lost, the SN dopamine neurons, rendering the whole system ineffective. The mechanism underlying this process is the major question in PD research. Is the primary site of injury the cell body, or perhaps the axon terminal, located in the striatum? Does the inflammatory response in the SN cause neuronal death or result from neuronal death? How do the remaining DA neurons and their targets in the striatum compensate for the loss of SN neurons? A functional system requires functional neurons, at both ends, and, to work properly, those neurons all need to execute the correct gene transcription and protein translation programs. Thus, monitoring of gene expression patterns in the nigrostriatal system can alert us to any abnormalities in the execution of the programs during PD. These molecular changes will provide information that is relevant to the functionality of the nigrostriatal system as a whole and perhaps point out candidate therapeutic targets and biomarkers of disease progression that can benefit patients directly (FIG. 1C).

Our approach is based on several observations. First, PD caused by mutation or overexpression of $\alpha$-synuclein, MPTP intoxication, and idiopathic PD (of unknown cause) are clinically and pathologically indistinguishable, suggesting a common pathological cascade. ${ }^{1}$ Second, PD has a long duration, with molecular and cellular pathology preceding clinical signs for at least several years, and perhaps decades. It is therefore likely that cell autonomous factors mediate cell death in PD. Understanding these factors at the earliest stages of disease may aid in the diagnosis and therapeutic approach to PD. Third, PD primarily targets the dopamine 
neurons of the SN for cell death, resulting in DA depletion in the striatum. The impact of injury in both of these regions (and perhaps others) should be assessed to understand the degradation of the nigrostriatal projection. Thus, a systems-based analysis of PD must encompass more than one etiology, span a range of disease stages, from preclinical to symptomatic, and examine the whole nigrostriatal pathway (FIG. 1B). Microarray analysis of gene expression changes is an optimal tool to apply to such a system.

\section{GENE EXPRESSION ANALYSIS}

Microarrays were developed in the mid-1990s and have quickly become the dominant method for mRNA level assessment on a large scale. To perform microarray analysis, one must extract RNA from a tissue of interest. Depending on the goals of the project, RNA may be obtained from a heterogeneous population of cells, such as from the $\mathrm{SN}$, or from single cells defined by one or more phenotypic feature, such as tyrosine hydroxylase (TH) positivity (see, for example, Ginsberg and cowork$\mathrm{ers}^{11}$ in this issue). The RNA is reverse transcribed to produce cDNA, which is subsequently in vitro transcribed into cRNA, incorporating a label that will permit its later quantitation on the array [for CodeLink bioarrays (GE Healthcare) and Affymerix GeneChips, the label is biotin]. cRNA is fragmented in preparation for hybridization to the array. Hybridization occurs between the labeled cRNA from the biological sample and the oligonucleotide probes on the array (probes are either covalently attached to the chip or synthesized directly on the chip; see Ginsberg and coworkers ${ }^{11}$ and Olson ${ }^{12}$ in this issue for additional discussion). The amount of RNA present in the original sample is estimated from the fluorescence intensity generated by hybridization with the complementary probe. Numerous commercial arrays are available for every species, many with the entire genome represented. Despite initial skepticism over quality, cost, and the relative paucity of statistical power to analyze such large datasets, the technology has continually met demands for improvement, while sophisticated statistical algorithms for normalization and data analysis have relieved some (but not all) concerns that microarrays are mere fishing expeditions in which one is just as likely to pull out false candidates as the real thing (reviewed in Yakovlev and coworkers ${ }^{13}$ and Olson, ${ }^{12}$ this issue, and Fathallah-Shaykh ${ }^{14}$ ). As microarrays become even more reliable and cost effective, their utility in investigating pathogenic cascades in ND should be fairly universal. Here we review the experience with microarray analysis applied to PD and PD model systems, but it should be noted that the systematic analysis of human patients and model systems for $\mathrm{AD},{ }^{15-17}$ amyotrophic lateral sclerosis, ${ }^{18,19}$ and Huntington's disease (HD), ${ }^{20-22}$ among other ND, is also underway. A detailed comparison of gene expression profiles between PD and other ND, though of great interest, is beyond the scope of this review.

\section{TOXIN-INDUCED MODELS OF PD}

Several studies have analyzed gene expression in MPTP-treated mice. The earliest data were generated from cDNA arrays carrying only about 1000 gene probes. ${ }^{23}$ The authors identified a number of gene expression changes associated with subchronic and acute MPTP intoxication, many of which were normalized by cotreatment with $R$-apomorphine, a neuroprotective factor in their model. ${ }^{23}$ Dominant categories of gene expression changes included oxidative stress, inflammation, and glutamate neurotransmission. The RNA used in these early studies was obtained from a limited number of mice and was derived from a mixture of cells from $\mathrm{SN}$, striatum, and other subcortical regions. Though the gene expression changes in the Youdim studies ${ }^{23}$ were not independently validated, more recent reports have found many of the same functional categories affected in MPTP models of PD.

A slightly different subchronic MPTP dosing paradigm was used to examine gene expression changes over time in a preclinical model of PD. In this model, mice are asymptomatic, but lose $35 \%$ of the $\mathrm{TH}$-positive neurons in the SN by 7 days following the final MPTP dose, the later time point in the study. ${ }^{24}$ Gene expression was assessed from carefully microdissected $\mathrm{SN}$ and striatum at 1 and 7 days after the completion of MPTP injections. This approach allows the observer to assess molecular events at the site of injury (SN) and its target (striatum) as the injury evolves over time. Interestingly, the SN showed many gene expression changes at the early time point and fewer at the later time point, suggesting a partial return to baseline. ${ }^{24}$ In contrast, the striatum showed a small number of gene expression changes at the early time point, but a quite large transcriptional response 1 week after the injections, suggesting a delayed reaction to nigral cell demise. ${ }^{25}$ This pattern was stable across two different array platforms used in the studies (GE CodeLink and Affymetrix). In both regions, the most notable classes of genes that were altered by MPTP were synaptic, cytoskeletal, and adhesion molecules and were neurotransmitter related. Mitochondrial genes and inflammatory/glial genes were also prominent in the SN data set. Both studies of MPTP-treated mice also report limited changes in the ubiquitin-proteasome system, cell cycle regulators, and oxidative stress response. Importantly, unsupervised clustering analysis of both $\mathrm{SN}$ and striatum tissue samples by gene expression level readily segregated the MPTP-treated from control samples, suggesting that the differentially expressed 
genes are directly relevant to disease status. ${ }^{24,25}$ For the MPTP-treated SN, clustering analyses indicated that the late MPTP samples were closer to controls than the early MPTP samples, consistent with our interpretation of the reduced number of expression changes at the later time point.

A related study of 6-hydroxydopamine (6-OHDA) toxicity identified a number of gene expression changes in the Parkinsonian rat striatum. ${ }^{26}$ 6-OHDA is a particularly potent toxin for catecholaminergic neurons in the rat midbrain, resulting in near complete depletion of DA in the striatum. ${ }^{27,28}$ Genes that were dysregulated following 6-OHDA lesions were interpreted to participate in the conveyance of the DA signal from the $\mathrm{SN}$ to the striatum. Furthermore, several genes that were altered are predicted to regulate the strength of glutamateric signaling in the striatum. These findings seem generally consistent with those from MPTP-treated mice (though formal comparisons have not been done to our knowledge) and support earlier models whereby loss of DA in the striatum is compensated for by alteration in GABAergic and glutamateric systems during PD progression. Importantly, this model extends the microarray data set to another species and another DA neuron toxicant.

Finally, microarrays have been used to look at gene expression profiles in cultured cell models of DA neuron death, systems that have the great advantage of homogeneity. Two unique midbrain-derived DA neuron cell lines (MN9D and SN4741) were treated with the MPTP derivative, MPP + , to invoke moderate cell death. The MN9D study found significant regulation of the endoplasmic reticulum-mediated unfolded protein stress response (UPR), including upregulation of $\mathrm{CHOP} / \mathrm{Gadd}$ 153 , a stress-induced transcription factor, by both MPP+ and 6-OHDA. ${ }^{29}$ Confirmatory protein analysis of these cells found post-translational modifications associated with the UPR, such as PERK and eIF $2 \alpha$ phosphorylation, also occurred after both toxin treatments. Microarray analysis of SN4741 cells identified other oxidative stress-induced genes, including several mitochondrial electron transport chain subunits, vesicle transport genes such as syntaxin 8 , and the Lewy body component, heme oxygenase- $1 .{ }^{30,31}$ While cell culture models can provide high signal-to-noise ratios in microarray experiments, facilitating the identification of differentially regulated genes, the cells are not postmitotic and lack contextual cues found in the intact nigrostriatal system, which may affect their transcriptional responses to DA toxins.

\section{GENETIC MODELS OF PD AND MICROARRAY STUDIES}

Despite the widespread availability of transgenic mouse models of PD, most commonly carrying $\alpha$-synuclein mutations, there is a scarcity of microarray data from genetic models of PD. The first analysis of gene expression in an $\alpha$-synuclein transgenic model was performed in flies. Comparing the gene expression profiles of flies transgenically expressing human $\alpha$-synuclein to age-matched tau-expressing transgenics, a unique signature for $\alpha$-synuclein-mediated degeneration was defined. ${ }^{32}$ The study included the entire Drosophila genome and examined multiple time points, from presymptomatic through end stage disease. Importantly, many of the approximately 50 genes that were dysregulated were altered at the very earliest time points, indicating that changes in genes related to lipid, membrane transport, and energy homeostasis were correlated with $\alpha$-synuclein expression before the onset of degeneration. ${ }^{32}$ This study stands out for its elegant design and straightforward analysis. The value of model organisms in dissecting critical features of the pathogenesis in PD should not be overlooked. As data from mice and other $\alpha$-synuclein model organisms become available, it will be useful to compare those gene expression profiles with the fly signature.

To our knowledge, there are no published reports of gene expression analysis using microarrays in $\alpha$-synuclein transgenic mice. Our lab has recently examined gene expression in three different transgenic mouse lines (Miller et al., unpublished data). As with earlier studies, we found that gene expression changes occurring at very early stages of disease in the $\mathrm{SN}$ (but not striatum in these mice) were capable of correctly predicting disease status, even in older animals, as shown by unsupervised clustering. There are many transgenic $\alpha$-synuclein mouse lines (reviewed in Fleming et al. ${ }^{9}$ ), each with strengths and weaknesses. The three lines used in this study carried different human $\alpha$-synuclein transgenes under control of different promoters and modeled different stages and severity of disease. Significantly differentially expressed genes were identified and validated using quantitative RT-PCR (Miller and Federoff, unpublished data).

\section{IDIOPATHIC HUMAN PD AND MICROARRAY STUDIES}

The standard for judging any model system is the human condition it emulates. In the case of PD, both toxicant and genetic models faithfully capitulate many of the cardinal pathologic features of disease, though no one model is perfect. Aided by the many generous patients and families who elect to donate their brains for research, several analyses of gene expression in postmortem PD tissues have been completed. ${ }^{33-35}$ Each of these studies identified a unique PD-associated molecular profile, with many of the same themes recurring. For instance, genes related to DA function 

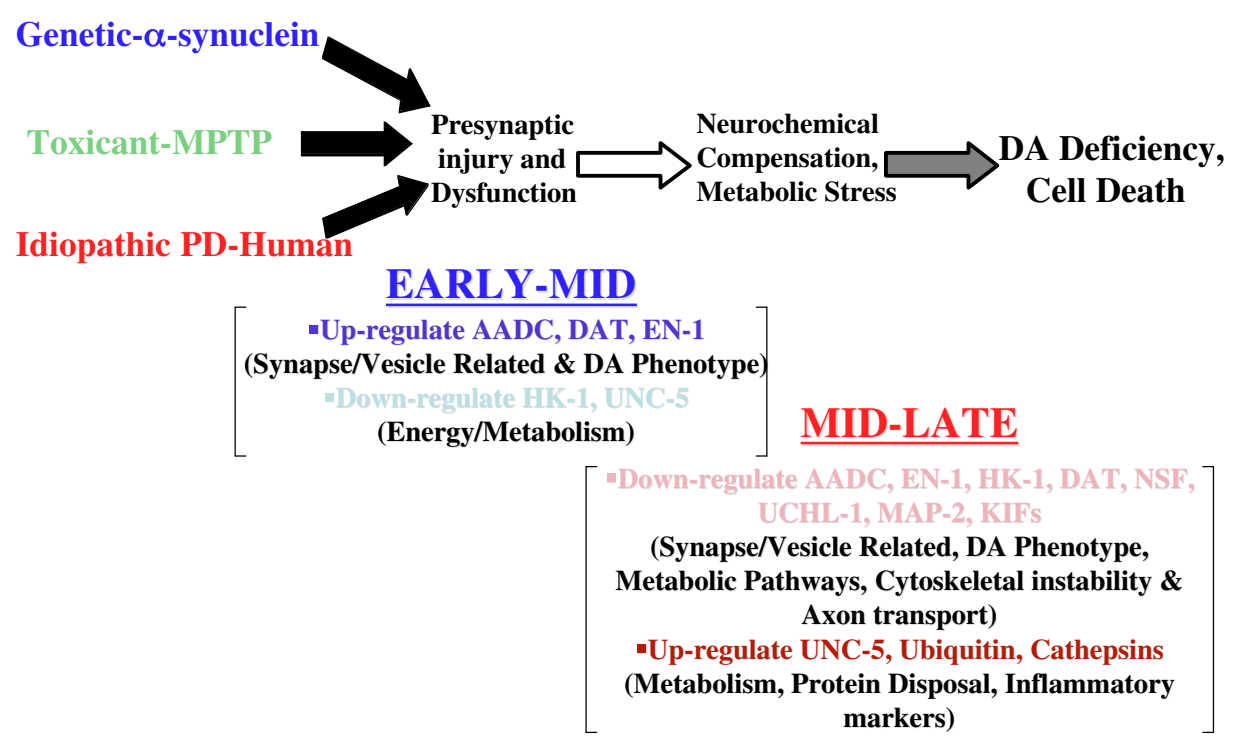

FIG. 2. Common pathways to pathogenesis revealed by gene expression analysis. Parkinson's disease may be caused by genetics, environment, or an unknown combination of genetic risk factors and environmental insults. Each stage in the cascade is hypothesized to exhibit a unique pattern of gene expression, or profile, which can be ascertained via high-throughput microarray analysis. The earliest event is likely to be damage to SN dopaminergic neurons, possibly at the presynaptic terminal. Gene expression changes that are typical of this early, preclinical disease phase (blue) are shown below (prominent increase in genes related to synapses and DA phenotype). Cell autonomous dysfunction in the SN will lead to DA depletion in the striatum, the major cause of phenotypic symptoms. A period of neurochemical and antioxidant-mediated compensation ensues, but is ultimately unable to prevent massive DA neuronal loss and clinical disease. Later disease stages, when clinical symptoms such as rigidity, tremor, and bradykinesia are apparent (red), are characterized by prominent decreases in genes related to the DA phenotype, synapses, cytoskeletal stability, and axonal transport (shown below). Gene expression changes are coded by pale color (decreased expression) or dark color (increased expression).

are downregulated, along with chaperones, ubiquitin proteasome subunits, and synaptic genes. Massive impairment in synaptic gene expression in both SN and striatum echoed that seen in MPTP-treated mice. ${ }^{35}$ Frequent changes in mitochondrial genes ${ }^{33}$ and oxidative stress $^{34}$ were also observed. Unsupervised clustering was used to segregate samples by gene expression levels in two of the studies, with perfect segregation of both SN and striatum tissues from control and PD samples in one study ${ }^{35}$ and nearly complete segregation of PD (4 of 6), frontotemporal dementia with parkinsonism (1 of 1 ), and progressive supranuclear palsy ( 2 of 2 ) from five controls. ${ }^{33}$ The ability for significantly differentially expressed genes to isolate tissue samples according to their disease state indicates that those genes' expression levels are informative. Particularly in a human population, differential gene expression could theoretically arise from differences in genetic background, gender, age, race, education, or geographical location (to name a few). If this were the case, significantly different genes might be predicted to segregate samples according to one or more of these features, in addition to disease state, thereby blurring the line between controls and PD samples. This is generally not the case, indicating that, for the regions examined, the genes that are altered are related to PD pathogenesis.

\section{APPLYING THE SYSTEMS-BASED APPROACH TO PD}

Alone, each model system and idiopathic PD-derived microarray data set has great utility in the search for pathogenic mechanisms in this disease. The highest value, however, is likely to be in the meta-analysis of gene expression changes across systems and array types, at different stages of disease, and in different brain regions. Together, toxin and genetic models with human PD cases can span the known causes of disease, model selectively early (preclinical) or late (symptomatic) disease stages, and provide unique opportunities to compare primary injury sites with downstream target regions (FIG. 2). Comparing gene expression patterns across arrays is also possible, facilitating cross-analysis and data sharing between laboratories using different array platforms. Extending the assumption that, because clinical and pathological PD phenotype is invariant despite variable known causes, a common pathogenic cascade is operative during disease, we believe that gene expression changes central to pathogenesis ought to be conserved among disease systems. To test this hypothesis, we have performed limited comparison of gene expression changes in human PD, MPTP-treated mice, and $\alpha$-synuclein transgenic mice. Ideally, such an analysis should consider gene expression changes reported from 
TABLE. Shared Gene Expression Changes in Three Models of PD

\begin{tabular}{lccc}
\hline Gene Name & $\begin{array}{c}\alpha \text {-syn* } \\
\text { (early) }\end{array}$ & $\begin{array}{c}\text { MPTP } \\
\text { (mid-late) }\end{array}$ & $\begin{array}{c}\text { Human } \\
\text { PD }^{\ddagger} \text { (late) }\end{array}$ \\
\hline ZFP-162 & $\Uparrow$ & $\Uparrow$ & $\Uparrow$ \\
UNC-5 & $\Downarrow$ & $\Uparrow$ & $\Uparrow$ \\
EN-1 & $\Uparrow$ & $\Downarrow$ & $\Downarrow$ \\
DAT & $\Uparrow$ & $\Downarrow$ & $\Downarrow$ \\
HK-1 & $\Downarrow$ & $\Downarrow$ & $\Downarrow$ \\
DSCR1-L1 & $\Downarrow$ & $\Downarrow$ & $\Downarrow$ \\
\hline
\end{tabular}

*Gene expression changes in the SN relative to nontransgenic controls at 3 or 6 months of age (early stage disease). ${ }^{\dagger}$ Gene expression changes in the $\mathrm{SN}$ relative to saline-treated controls at $24 \mathrm{~h}$ or 7 days after the final dose of MPTP (mid-late stage disease). ${ }^{\ddagger}$ Gene expression changes relative to nondisease control SN (late stage disease).

as many different models and PD patients as possible, and it is our hope that, with the free and open sharing of microarray datasets and increasingly sophisticated statistical tools, this will become a reality in the near future. Based on their neuropathological characterization, we have assigned each model system used in our studies a disease stage category. $\alpha$-synuclein transgenic mice (THsynWT) show no cell loss and mild neurochemical phenotypes at the ages used ( 3 and 6 months: early stage). MPTP-treated mice show 35\% DA neuron loss and nearly complete loss of DAT expression in the striatum, but are asymptomatic, and we assign them as mid-late stage disease. All of the human PD subjects in our studies were clinically and pathologically late stage disease. Significant gene lists from analysis of the SN in each of the three models (MPTP, $\alpha$-synuclein, and human PD) were reduced to the CodeLink Probe ID number and loaded into File Maker Pro to create a searchable database of differentially expressed genes. We queried this database to identify Probe IDs that were present in all three of the lists. Our analysis identified a unique set of six genes that are differentially expressed compared with controls in all three disease systems. ${ }^{36}$ These genes were the DAT, engrailed homolog 1 (EN-1), uncoupling protein 5 (UNC-5), hexokinase 1 (HK-1), zinc finger protein 162 (ZFP 162), and Down's syndrome related protein 1-like 1 (DSCR1-11). Intriguingly, we did not observe the same expression profile for each of these genes in all three models (Table 1). Some of the genes were increased at presymptomatic stages of disease ( $\alpha$-synuclein mice) and decreased in disease stages at which significant cell loss had occurred (MPTP mice and human PD). For instance, we see that the two genes linked to the DA phenotype, DAT and EN-1, are both upregulated in $\alpha$-synuclein mice, which are considered early stage disease, but significantly reduced in both MPTP and human PD. Thus, the DA neurons exposed to high concentrations of $\alpha$-synuclein might engineer a strategy to protect from what is perceived early on as a toxic threat, by enhancing selected phenotypic properties. In contrast, the ravages of PD and MPTP intoxication result in loss of these properties, rendering susceptible neurons more likely to succumb to pathogenesis. The mitochondrial uncoupling gene, UNC-5, has the opposite expression pattern. It is decreased in $\alpha$-synuclein mice and upregulated in human PD and MPTP-treated mice. UNC-5 may enhance proton leak from the inner mitochondrial membrane but is also important in reactive oxygen species scavenging. ${ }^{37}$ Whether this alteration would be protective or harmful in the different systems is not entirely clear. HK-1 and DSCR1-11 are decreased in all groups, indicating that they may be predictive of disease even at the earliest stages. HK-1 has been found to be associated with mitochondria in axons, raising the possibility that loss of HK-1 early in axon terminals may trigger presynaptic dysfunction by altering energy levels. DSCR1-11 is relatively unstudied, but may act similarly to its relative, DSCR1. Both inhibit calcinuerin, thereby prolonging calcium-dependent signaling events. DSCR1 was recently shown to regulate the expression of several genes, including superoxide dismutase and $\mathrm{TH}$, which have both been implicated in PD. ${ }^{38} \mathrm{ZFP}-162$, in contrast to the other genes, was increased in all three groups. This gene could potentially have many pleiotropic effects, based on its role as a STAR family member (signal transduction and RNA binding). Our limited analysis has yielded some familiar and some new candidates that warrant further attention.

One gene that we are particularly interested in is EN-1. EN-1 is required for the development of DA neurons and has recently been shown to play a role in the proper innervation of the striatum by DA axons. ${ }^{39,40}$ Moreover, EN-1 regulates the expression of $\alpha$-synuclein in vivo. ${ }^{39}$ Its interesting changes in expression at different stages of PD (increased early on, decreased at later time points) indicate that it may be important for maintaining the viability of DA neurons during disease-causing injury. For example, increasing levels of EN-1 may help protect DA neurons initially, but eventually, pathogenic pathways dominate, resulting in reduced levels of EN-1 coincident with neuronal demise. If studies currently underway indeed validate the importance of EN-1 in PD pathogenesis, this gene would make an excellent target for therapeutic intervention. The expression pattern of $\mathrm{EN}-1$ is spatially restricted during development to the mid-hindbrain border and throughout adulthood to the $\mathrm{SN}$ and ventral tegmental area, making it relatively safe to target with minimal nonspecific effects. Alternatively, achieving a better understanding of EN-1 transcriptional targets may yield even more selective targets for PD therapy. For example, if high EN-1 levels are protective because transcription of target gene $\mathrm{X}$ is enhanced, directly upregulating $X$ may be even more effective than 
altering EN-1 expression. Though these possibilities are purely speculative at this point, they highlight the potential of microarray analysis for ND and neurotherapeutics.

\section{CONCLUSION}

Applying the microarray approach to additional disease models and many more human samples is required to obtain a gene expression signature of PD progression. However, with the current datasets, there is still much that can be done. For example, the application of alternative data mining techniques to existing data is predicted to give new significant gene lists (see, for example, the fusion strategy reviewed in Miller and Federoff ${ }^{36}$ ). The true alterations should appear on all lists, regardless of the analysis method used, and as algorithms become more sophisticated, we expect to see less variability in this respect (Olson, ${ }^{13}$ this issue). Going beyond regional gene expression changes, evaluating profiles of single cells or single cell types from PD brains is also possible (Ginsberg and coworkers, ${ }^{11}$ this issue). This would take the analysis down another level and, though technically challenging, should reduce much of the noise in the data arising from nonspecific sources (such as non-DA neurons and glial cells resident in the $\mathrm{SN})$. Additionally, useful profiles could be obtained from those other distinct cell populations, GABAergic neurons in the SN pars reticulata and astrocytes resident in the $\mathrm{SN}$ or striatum, elucidating their transcriptional responses to injured DA neurons. Others are interested in profiling gene expression in peripheral tissues such as fibroblasts and lymphocytes. It is becoming more accepted to view ND as systemic disorders, with primary sites of injury occurring in the CNS and surrogate or secondary effects in the periphery. Monitoring peripheral gene expression patterns during disease has several potential advantages. First, the tissue is readily accessible, allowing multiple samplings over time. Second, normalization of abnormal expression associated with disease (or failure to normalize) after treatment would provide a quantitative measurement of the efficacy of the treatment. Third, genome profiling, including analysis of single nucleotide polymorphisms (SNPs), in peripheral tissue may aid in the discovery of genetic susceptibility factors for disease, as well as inform the physician's choice of treatments based on an individual genotype. These possibilities may seem unrealistic now, but many of these methods are becoming routine in the treatment of certain cancers, and certainly the age of individualized medicine is not far off. A movement is already underway to compare gene expression data between diseases to ascertain disease-specific pathways versus secondary effects seen in multiple ND. For example, collaborative efforts to compare the gene expression profiles of PD and HD striatum samples are underway (A. Strand, RM Miller, and HJ Federoff, un- published observations). This and similar efforts highlight the open data-sharing environment among those interested in understanding molecular cascades in ND. Collaboration between labs and concerted efforts aimed at pooling and sharing microarray datasets from PD and other ND should continue to increase as more data are generated. Eventually, microarray technology may progress to the point where it can aid in the diagnosis of ND and monitor an individual's response to therapeutic intervention over time. In the short term, new hypotheses are being generated and specific pathogenic molecular models of dysfunction based on novel candidate genes can be tested.

\section{REFERENCES}

1. Maguire-Zeiss KA, Federoff HJ. Convergent pathobiologic model of Parkinson's disease. Ann NY Acad Sci 991:152-166, 2003.

2. Bezard E, Gross CE, Fournier MC, Dovero S, Bloch B, Jaber M. Absence of MPTP-induced neuronal death in mice lacking the dopamine transporter. Exp Neurol 155:268-273, 1999.

3. Gainetdinov RR, Fumagalli F, Jones SR, Caron MG. Dopamine transporter is required for in vivo MPTP neurotoxicity: evidence from mice lacking the transporter. J Neurochem 69:1322-1325, 1997.

4. Gainetdinov RR, Fumagalli F, Wang YM, Jones SR, Levey AI, Miller GW, et al. Increased MPTP neurotoxicity in vesicular monoamine transporter 2 heterozygote knockout mice. J Neurochem 70:1973-1978, 1998.

5. Teismann P, Tieu K, Cohen O, Choi DK, Wu du C, Marks D, et al. Pathogenic role of glial cells in Parkinson's disease. Mov Disord 18:121-129, 2003.

6. Perez FA, Curtis WR, Palmiter RD. Parkin-deficient mice are not more sensitive to 6-hydroxydopamine or methamphetamine neurotoxicity. BMC Neurosci 6:71, 2005.

7. Fornai F, Schluter OM, Lenzi P, Gesi M, Ruffoli R, Ferrucci M et al. Parkinson-like syndrome induced by continuous MPTP infusion: convergent roles of the ubiquitin-proteasome system and alpha-synuclein. Proc Natl Acad Sci USA 102:3413-3418, 2005.

8. Galvin JE, Lee VM, Trojanowski JQ. Synucleinopathies: clinical and pathological implications. Arch Neurol 58:186-190, 2001.

9. Fleming SM, Fernagut PO, Chesselet MF. Genetic mouse models of parkinsonism: strengths and limitations. NeuroRx 2:495-503, 2005.

10. Bove J, Prou D, Perier C, Przedborski S. Toxin-induced models of Parkinson's disease. NeuroRx 2:484-494, 2005.

11. Ginsberg SD, Che S, Counts SE, Mufson EJ. Single cell gene expression profiling in Alzheimer's disease. NeuroRx 3:302-317, 2006.

12. Olson NE. The microarray data analysis process: from raw data to biological significance. NeuroRx 3:371-381, 2006.

13. Almudevar A, Klebanov LB, Qiu X, Salzman P, Yakovlev AY. Utility of correlation measures in analysis of gene expression. NeuroRx 3:382-393, 2006.

14. Fathallah-Shaykh HM. Microarrays: applications and pitfalls. Arch Neurol 62:1669-1672, 2005.

15. Blalock EM, Chen KC, Stromberg AJ, Norris CM, Kadish I, Kraner SD et al. Harnessing the power of gene microarrays for the study of brain aging and Alzheimer's disease: statistical reliability and functional correlation. Aging Res Rev 4:481-512, 2005.

16. Colangelo V, Schurr J, Ball MJ, Pelaez RP, Bazan NG, Lukiw WJ. Gene expression profiling of 12633 genes in Alzheimer hippocampal CA1: transcription and neurotrophic factor down-regulation and up-regulation of apoptotic and pro-inflammatory signaling. J Neurosci Res 70:462-473, 2002.

17. Loring JF, Wen X, Lee JM, Seilhamer J, Somogyi R. A gene expression profile of Alzheimer's disease. DNA Cell Biol 20:683$695,2001$. 
18. Dangond F, Hwang D, Camelo S, Pasinelli P, Frosch MP, Stephanopoulos G, et al. Molecular signature of late-stage human ALS revealed by expression profiling of postmortem spinal cord gray matter. Physiol Genomics 16:229-239, 2004.

19. Jiang YM, Yamamoto M, Kobayashi Y, Yoshihara T, Liang Y, Terao S, et al. Gene expression profile of spinal motor neurons in sporadic amyotrophic lateral sclerosis. Ann Neurol 57:236-251, 2005.

20. Chan EY, Luthi-Carter R, Strand A, Solano SM, Hanson SA, DeJohn MM, et al. Increased huntingtin protein length reduces the number of polyglutamine-induced gene expression changes in mouse models of Huntington's disease. Hum Mol Genet 11:19391951, 2002.

21. Borovecki F, Lovrecic L, Zhou J, Jeong H, Then F, Rosas HD, et al. Genome-wide expression profiling of human blood reveals biomarkers for Huntington's disease. Proc Natl Acad Sci USA 102: 11023-11028, 2005.

22. Strand AD, Aragaki AK, Shaw D, Bird T, Holton J, Turner C, et al. Gene expression in Huntington's disease skeletal muscle: a potential biomarker. Hum Mol Genet 14:1863-1876, 2005.

23. Grunblatt E, Mandel S, Maor G, Youdim MB. Gene expression analysis in $N$-methyl-4-phenyl-1,2,3,6-tetrahydropyridine mice model of Parkinson's disease using cDNA microarray: effect of $R$-apomorphine. J Neurochem 78:1-12, 2001.

24. Miller RM, Callahan LM, Casaceli C, Chen L, Kiser GL, Chui B, et al. Dysregulation of gene expression in the 1-methyl-4-phenyl1,2,3,6-tetrahydropyridine-lesioned mouse substantia nigra. $J$ Neurosci 24:7445-7454, 2004.

25. Miller RM, Chen LL, Kiser GL, Giesler TL, Kaysser-Kranich TM, Palaniappan C, et al. Temporal evolution of mouse striatal gene expression following MPTP injury. Neurobiol Aging 26:765-775, 2005.

26. Napolitano M, Centonze D, Calce A, Picconi B, Spiezia S, Gulino A, et al. Experimental parkinsonism modulates multiple genes involved in the transduction of dopaminergic signals in the striatum. Neurobiol Dis 10:387-395, 2002.

27. Schwarting RK, Huston JP. Unilateral 6-hydroxydopamine lesions of meso-striatal dopamine neurons and their physiological sequelae. Prog Neurobiol 49:215-266, 1996.

28. Schultz W. Depletion of dopamine in the striatum as an experimental model of Parkinsonism: direct effects and adaptive mechanisms. Prog Neurobiol 18:121-166, 1982.
29. Holtz WA, O’Malley KL. Parkinsonian mimetics induce aspects of unfolded protein response in death of dopaminergic neurons. J Biol Chem 278:19367-19377, 2003

30. Yoo MS, Chun HS, Son JJ, DeGiorgio LA, Kim DJ, Peng C, et al. Oxidative stress regulated genes in nigral dopaminergic neuronal cells: correlation with the known pathology in Parkinson's disease. Brain Res Mol Brain Res 110:76-84, 2003.

31. Chun HS, Gibson GE, DeGiorgio LA, Zhang H, Kidd VJ, Son JH. Dopaminergic cell death induced by $\operatorname{MPP}(+)$, oxidant and specific neurotoxicants shares the common molecular mechanism. J Neurochem 76:1010-1021, 2001.

32. Scherzer CR, Jensen RV, Gullans SR, Feany MB. Gene expression changes presage neurodegeneration in a Drosophila model of Parkinson's disease. Hum Mol Genet 12:2457-2466, 2003.

33. Hauser MA, Li YJ, Xu H, Noureddine MA, Shao YS, Gullans SR et al. Expression profiling of substantia nigra in Parkinson's disease, progressive supranuclear palsy, and frontotemporal dementia with parkinsonism. Arch Neurol 62:917-921, 2005.

34. Grunblatt E, Mandel S, Jacob-Hirsch J, Zeligson S, Amariglo N, Rechavi G, et al. Gene expression profiling of parkinsonian substantia nigra pars compacta: alterations in ubiquitin-proteasome, heat shock protein, iron and oxidative stress regulated proteins, cell adhesion/cellular matrix and vesicle trafficking genes. J Neural Transm 111:1543-1573, 2004.

35. Miller RM, Kiser GL, Kaysser-Kranich TM, Lockner RJ, Palaniappan C, Federoff HJ. Robust dysregulation of gene expression in substantia nigra and striatum in Parkinson's disease. Neurobiol Dis 21:305-313, 2006.

36. Miller RM, Federoff HJ. Altered gene expression profiles reveal similarities and differences between Parkinson's disease and model systems. Neuroscientist 11:539-549, 2005.

37. Erlanson-Albertsson C. Uncoupling proteins: a new family of proteins with unknown function. Nutr Neurosci 5:1-11, 2002.

38. Ermak G, Cheadle C, Becker KG, Harris CD, Davies KJ. DSCR1(Adapt78) modulates expression of SOD1. FASEB $J$ 18: 62-69, 2004

39. Simon HH, Saueressig H, Wurst W, Goulding MD, O'Leary DD. Fate of midbrain dopaminergic neurons controlled by the engrailed genes. J Neurosci 21:3126-3134, 2001.

40. Simon HH, Bhatt L, Gherbassi D, Sgado P, Alberi L. Midbrain dopaminergic neurons: determination of their developmental fate by transcription factors. Ann NY Acad Sci 991:36-47, 2003. 